inflammatory/cytotoxic characteristics. These changes clearly impacted tumor cell phenotype, as cells from the scaffold increased tumor cell migration and apoptosis in vitro. DISCUSSION/ SIGNIFICANCE OF IMPACT: Early phenotypic changes at the engineered metastatic niche can identify signs of metastasis prior to colonization of tumor cells. Furthermore, dynamics of immune and stromal cells change throughout niche maturation, influencing tumor cell phenotype and may suggest targeted therapies. CONFLICT OF INTEREST DESCRIPTION: Lonnie Shea, Jacqueline Jeruss, and Grace Bushnell are named inventors on patents or patent applications.

4579

Distinct clinical and immunological responses to $\alpha$ PD-1, k $\alpha$ PD-L1 and $\alpha$ PD-L2 immunotherapy in B16 melanoma in aged versus young hosts includes T-cell stem cell effects and PD-L2 expression differences

Myrna G Garcia ${ }^{1}$, Alvaro Padron, Yilun Deng, Harshita Gupta, Aravind Kancharla, Ryan Reyes, and Tyler Curiel

${ }^{1}$ University of Texas Health Science Center San Antonio

OBJECTIVES/GOALS: Aging is the biggest risk factor for cancer, yet little is known about cancer immunotherapy effects. Here we investigate melanoma response to $\alpha \mathrm{PD}-1, \alpha \mathrm{PD}-\mathrm{L} 1$ and $\alpha \mathrm{PD}-\mathrm{L} 2$ in young vs. aged hosts. We look at different immune outcomes as possible mechanism. METHODS/STUDY POPULATION: We tested $\alpha \mathrm{PD}-1$ (100 $\mu \mathrm{g} /$ mouse), $\alpha$ PD-L1 (100 $\mu \mathrm{g} /$ mouse) or $\alpha$ PD-L2 (200 $\mu \mathrm{g} /$ mouse $)$ in aged (18-24 months) and young (3-8 months) mice challenged orthotopically with B16. Tumors and draining lymph nodes (TDLN) were analyzed by flow. Bone marrow-derived DC were generated with GM-CSF. RESULTS/ANTICIPATED RESULTS: We reported that $\alpha \mathrm{PD}-1$ treats young and aged with $\mathrm{B} 16$ and $\alpha \mathrm{PD}-\mathrm{L} 1$ only treats young. aPD-L2 treated B16 in aged but, remarkably, not young, the first anti-cancer single agent immunotherapy exhibiting this property. Efficacy in young (aPD-1, aPD-L1) and aged (aPD-1, aPD-L2) correlated with increased T cell stem cells (TCSC) and total tumorinfiltrating lymphocytes (TIL), but TCSC differed by age and treatment (e.g., distinct CCR2, CXCR5, CXCR3, PD-1 and TIM- expression). Aged expressed significantly more T-cell PD-1 and up to 40-fold more PD-L2 versus young in myeloid and NK cells, and TCSC. Bone marrow-derived DC experiments suggest aged DC are destined for high PD-L2 versus young. DISCUSSION/ SIGNIFICANCE OF IMPACT: Treatment differences in aged vs. young could depend on immune checkpoint or TCSC differences. We are now identifying mechanisms for increased PD-L2 and contributions to aPD-L2 efficacy in aged, and testing TCSC effects. Our work can improve cancer immunotherapy in aged hosts and further provide important insights even in young hosts.

4071

Dynamic Control of Tumor Vessels Augments Antitumor Responses

Emmanuel M Gabriel, Assistant Professor ${ }^{1}$, Deborah Bahr ${ }^{2}$, Sanjay Bagaria $^{2}$, Debrabata Muhkopadhyay ${ }^{2}$, and Keith Knutson ${ }^{2}$

${ }^{1}$ Mayo Clinic; ${ }^{2}$ Mayo Clinic Florida

OBJECTIVES/GOALS: Our overall objective is to develop a directly observable and reproducible method of enhanced blood flow through tumor vessels (i.e. dynamic control) at the time of systemic treatment delivery. Our central hypothesis is that the dynamic control of tumor vessels will improve (1) systemic drug delivery and (2) effector cell trafficking to target tumor. METHODS/STUDY POPULATION: B16 melanoma cells were inoculated into C57BL/6 (B6) mice (male and female) in both regional (hind leg) and systemic (flank) models. Dynamic control consisted of an IV saline bolus (500 ul) and phenylephrine (10 ug). Tumor vessel response was observed in real-time through window chambers using intravital microscopy (IVM). Dynamic control was combined with melphalan $(20 \mathrm{mg} / \mathrm{ml})$ either regionally (isolated limb perfusion) or systemically. Outcomes included tumor growth, survival, IHC, and toxicity. Dynamic control will be combined with adoptive transfer of effector T cells. B6 mice will be inoculated with B16/OVA (flank with window chamber) and treated with fluorescently labeled (calcein), OVA-specific CD8+ T cells from OT-1 transgenic mice. IVM, IHC, and flow cytometry will be used to measure $\mathrm{T}$ cell trafficking. RESULTS/ANTICIPATED RESULTS: Dynamic control (1) restored blood flow in non-functional tumor vessels and (2) increased and then transiently reversed blood flow in functional vessels. Vessel diameters did not change, suggesting that shunting of systemic blood to the tumor vasculature accounted for the observed changes. Dynamic control augmented tumor responses in our regional therapy model of melanoma. Increases in DNA adduct formation (melphalan mechanism of action) detected by IHC, decreased tumor growth, and increased survival were observed with dynamic control. There was no increased limb toxicity. Similarly, dynamic control augmented responses in our systemic therapy model (decreased tumor growth and improved survival). We anticipate that dynamic control will improve trafficking of effector T cells in the next set of experiments. DISCUSSION/ SIGNIFICANCE OF IMPACT: Heterogeneous responses to systemic therapies represent a major gap in current cancer treatment. An essential requirement for any effective therapy is its ability to reach tumor via the tumor-associated vasculature. We have therefore developed an approach to enhance drug delivery (dynamic control), which we also plan to test in clinical trials.

4578

\section{Early life stress promotes chronicity of experimental} colitis

Rachel Quinn Muir ${ }^{1}$, Barbara J. Klocke ${ }^{1}$, Kasi C. McPherson ${ }^{1}$, Jeremy B. Foote ${ }^{1}$, Jennifer S. Pollock ${ }^{1}$, and Craig L. Maynard ${ }^{1}$

${ }^{1}$ University of Alabama at Birmingham

OBJECTIVES/GOALS: The overall goal of this study was to determine the effect of early life stress (ELS) on the intestinal CD4+ T cell immune compartment, at homeostasis and after induction of experimental Inflammatory Bowel Disease (IBD). METHODS/STUDY POPULATION: We used a mouse model of ELS, maternal separation with early weaning (MSEW). We used IL-10 reporter mice to enable analysis of IL-10-producing cells. Mice were examined on postnatal day 28 to determine the impact of ELS on gut regulatory $\mathrm{T}$ cells. Plasma levels of corticosterone (rodent stress response hormone) was determined by ELISA. Colitis was induced in MSEW and normal rear (NR) mice via intraperitoneal injection of $\alpha$-IL-10R every 5 days until day 15. Mice were euthanized on days 20 and 30. Colonic tissue sections were stained for histological analysis. Remaining tissue was further processed for flow cytometric analysis of CD4+ T cells and innate lymphoid cells. RESULTS/ANTICIPATED RESULTS: Plasma corticosterone was elevated in MSEW mice compared to their NR counterparts at 4 weeks of age. We observed that the MSEW stress protocol does not affect the baseline colonic $\mathrm{CD} 4+\mathrm{T}$ cell or innate lymphoid cell populations. There was a reduction in the intestinal 
CD4 $+\mathrm{T}$ cells and regulatory T cells on day 20 in $\alpha$-IL-10R MSEW mice compared to NR counterparts. This difference disappeared by day 30 . Histological scoring showed no difference in disease severity between $\alpha$-IL-10R treated MSEW and NR mice on day 20. However, on day 30, when $\alpha$-IL-10R NR mice are recovering from colitis, MSEW mice showed persistent histological inflammation, mainly attributable to sustained epithelial damage. DISCUSSION/ SIGNIFICANCE OF IMPACT: Our results suggest that ELS prolongs intestinal inflammation and impairs epithelial repair. Future studies will focus on elucidating the mechanisms responsible for ELS-dependent impairment of mucosal repair in experimental colitis.

\section{Evaluating the effect of a compliant stent-graft prototype on effective stiffness in a cadaveric aorta} Shannen B Kizilski ${ }^{1}$, Filippo Coletti, $\mathrm{PhD}^{2}$, Rumi Faizer, $\mathrm{MD}^{2}$, and Victor H. Barocas, $\mathrm{PhD}^{2}$

${ }^{1}$ University of Minnesota CTSI; ${ }^{2}$ University of Minnesota

OBJECTIVES/GOALS: High aortic stiffness is associated with increased cardiovascular morbidity and mortality. The purpose of this work is to demonstrate the potential of our compliant stent-graft design to therapeutically increase aortic compliance over a standard aortic stent-graft. METHODS/STUDY POPULATION: The aorta from a human cadaver will be excised and placed into a pulse duplicator circuit. The stiffness of the system will be estimated using the pulse wave velocity (PWV), which will be calculated using the time delay between pressure measurements at proximal and distal locations in the system. Baseline measurements with the unstented aorta will be compared to two cases: (1) with a standard stent-graft placed, and (2) with our compliant stent-graft prototype in the descending thoracic aorta. PWV is calculated as the distance between the pressure sensors divided by the time delay. Faster PWV is associated with a stiffer vessel, or lower aortic compliance. RESULTS/ANTICIPATED RESULTS: Prior work in vitro showed that the compliant stent-graft reduced peak and pulse pressures compared a standard, rigid stent-graft. We also expect the compliant device to exhibit lower PWV compared to a rigid stent-graft. Depending on the aortic tissue stiffness, the compliant stent-graft could raise or lower PWV compared to no stent. Mean pressure in the compliant case is likely to be slightly higher than the other two cases because the compliant stent-graft's narrower lumen increases flow resistance. Although mean pressure will be higher, peak pressure should be lower than in the standard stent-graft because the added compliance decreases overall pressure swing between systole and diastole. DISCUSSION/SIGNIFICANCE OF IMPACT: Lower PWV in the compliant stent-graft over the standard stent-graft will indicate its potential to therapeutically lower aortic stiffness in patients needing aortic stenting. Positive outcomes from this study will be a step toward the eventual translation of a compliant stent-graft to clinical use.

4287

Extracellular vesicles as biomarkers for early detection of pancreatic cancer

Charles P Hinzman ${ }^{1}$, Shivani Bansal ${ }^{2}$, Yaoxiang $\mathrm{Li}^{2}$, Partha

Banerjee $^{2}$, and Amrita Cheema ${ }^{2}$

${ }^{1}$ Georgetown - Howard Universities; ${ }^{2}$ Georgetown University Medical Center

OBJECTIVES/GOALS: Pancreatic ductal adenocarcinoma (PDAC) is projected to become the second leading cause of cancer-related deaths by 2030. Though many other cancers have seen improvements in patient survival rates, patients diagnosed with PDAC have a 5-year survival rate of only $~ 9 \%$. A major contributor to decreased survival is late-stage diagnosis of the disease. New methods of early detection are urgently needed. Extracellular vesicles (EVs) are secreted from cells of all tissue types into the circulation. EVs play important roles in a variety of diseases. They have shown to promote cancer progression and they are being studied as potential biomarkers for disease diagnosis. The purpose of this study was to perform qualitative and quantitative characterization of smallmolecule profiles of EVs derived from various pancreatic cancer (PC) and normal pancreas cell lines, to provide proof-of-concept for evaluating the efficacy of leveraging EVs as potential biomarkers of PDAC. METHODS/STUDY POPULATION: EVs were isolated from the conditioned media of six PC and two normal pancreas cell lines using differential ultracentrifugation with filtration. EV enrichment was validated using quantitative ELISA, immunoblot and transmission electron microscopy. Targeted liquid chromatography coupled to mass spectrometry (LC-MS/MS) and untargeted (UPLC-QTOF-MS) metabolomics were used to analyze the biochemical composition of EVs. RESULTS/ANTICIPATED RESULTS: The biochemical profile of PC EVs was found to be significantly different from the profiles of normal cell EVs. Interestingly, amino acids were downregulated in PC EVs as compared to normal cell EVs. However, PC EVs were enriched in lactate and malate. PC EVs also had significant upregulation in other small molecules such as xanthosine, guanosine diphosphate and nicotinamide. DISCUSSION/SIGNIFICANCE OF IMPACT: Our results indicate that the biochemical characterization of EVs using metabolomics has the potential to yield biomarkers which can delineate cancer cell-derived EVs from normal cell-derived EVs. Further work will test the clinical significance of these findings by similar analyses of plasma of PDAC patients. Furthermore, these profiles may be detectable before progression of the disease to late-stage PDAC, leading to the development of assays for earlier diagnosis in patients.

4543

Glucocorticoid Receptors are essential effectors of TGF $\beta$ signaling in Triple Negative Breast Cancer

Carlos Jesus Perez Kerkvliet ${ }^{1}$, Amy R Dwyer, Caroline Diep, Robert Oakley, Christopher Liddle, and Carol A Lange

${ }^{1}$ University of Minnesota CTSI

OBJECTIVES/GOALS: The glucocorticoid receptor (GR) is a ubiquitous steroid hormone receptor that is emerging as a mediator of breast cancer metastasis. We aim to better understand the biology associated with phospho-GR species in TNBC and their contribution to tumor progression. METHODS/STUDY POPULATION: To better understand how p-S134 GR may impact TNBC cell biology, we probed GR regulation by soluble factors that are rich within the tumor microenvironment (TME), such as TGF $\beta$. TNBC cells harboring endogenous wild-type or S134A-GR species were created by CRISPR/Cas knock-in and subjected to in vitro assays of advanced cancer behavior. RNA-Seq was employed to identify pS134-GR target genes that are uniquely regulated by TGF $\beta$ in the absence of exogenously added GR ligands. Direct regulation of selected TGF $\beta$-induced $\mathrm{pS} 134$-GR target genes was validated accordingly. Bioinformatics tools were used to probe publicly available TNBC patient data sets for expression of a pS134-GR 24-gene signature. RESULTS/ANTICIPATED RESULTS: In the 